\title{
A Pore Scale Evaluation of the Kinetics of Mineral Dissolution and Precipitation Reactions (EMSI) (November 2006)
}

\author{
Carl I. Steefel
}

Lawrence Berkeley National Lab, Earth Sciences Division

\section{Overview and Research Objectives}

The chief goals for CEKA are to 1) collect and synthesize molecular-level kinetic data into a coherent framework that can be used to predict time evolution of environmental processes over a range of temporal and spatial scales; 2) train a cohort of talented and diverse students to work on kinetic problems at multiple scales; 3) develop and promote the use of new experimental techniques in environmental kinetics; 4) develop and promote the use of new modeling tools to conceptualize reaction kinetics in environmental systems; and 5) communicate our understanding of issues related to environmental kinetics and issues of scale to the broader scientific community and to the public.

The goal of the Berkeley Lab research effort (C.I. Steefel, PI) is to establish how laboratorydetermined reaction rates in (bio)geochemical systems relate to rates occurring in porous media under a variety of transport regimes. To do so, we are carrying out the first measurements of mineral-water reaction rates at the pore scale $(100-500 \mu \mathrm{m}$ pores) in microfluidic reactive flow devices and we are interpreting these rates with pore-scale reactive transport modeling. A second goal is to determine how weathering reactions increase porosity and pore connectivity at a weathering front, and whether this affects the rate of transport of reactive constituents to the front and thus the overall rate of chemical weathering.

\section{Research Progress}

New Experimental Techniques using Microfluidic Reactive Flow Devices

As a comparison with the baseline rates determined in batch and/or well-stirred flowthrough reactors (the typical approach in biogeochemistry), we are conducting pore-scale microfluidic reactive flow experiments, focusing initially on engineered pores containing calcite, Fe-oxide, and olivine.

\section{Reaction of bioreduced AQDS with hematite in a microfluidic cell}

Fe-oxide, which is deposited as a 20-30 nanometer thick coating on X-ray transparent silicon nitride windows using Molecular Beam Epitaxy (MBE), is reacted with the bioreduced electron shuttle AQDS $\left(\mathrm{AH}_{2} \mathrm{DS}\right)$ and reaction products are imaged with Scanning Transmission X-Ray Microscopy (STXM) at the Advanced Light Source (Beamline 11.0.2). Preliminary results showing the Fe L edge XANES spectrum for hematite reacted with bioreduced AQDS is shown in Figure 1. 


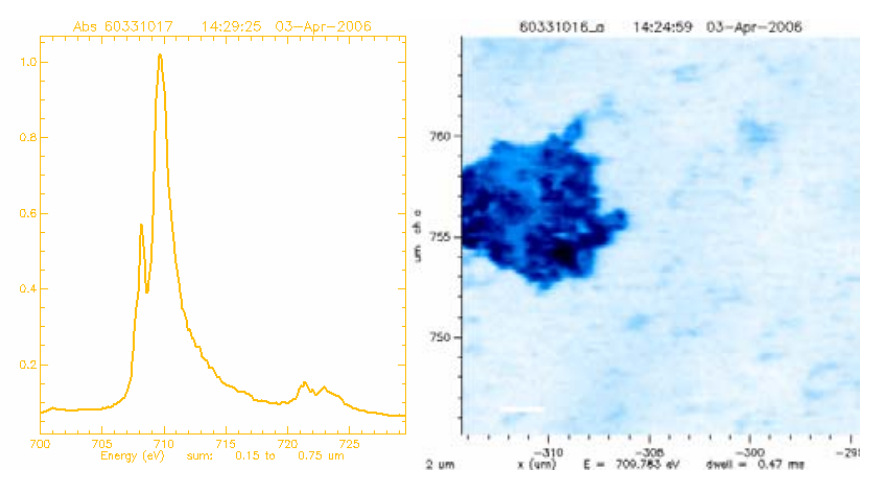

Figure 1: STXM results showing Fe L edge XANES spectrum for hematite reacted with bioreduced AQDS $\left(\mathrm{AH}_{2} \mathrm{DS}\right)$ in a microfluidic reactive flow cell.

\section{Calcite dissolution rates in single pores}

Microfluidic reactive flow experiments with calcite are being used to establish the comparison between rates determined in conventional laboratory flow-through reactors and rates determined in single pores. The experimental setup consists of a 500 micron thick wafer of pure calcite (Iceland spar) sandwiched between two ceramic supporting frames (Figure 2). A single cylindrical hole measuring 150 microns in diameter is drilled through the calcite and the supporting ceramic wafers. Inlet and outlet tubing is then connected to a low flow velocity syringe pump. Effluent total calcium concentrations, a measure of the extent of dissolution of the calcite in the pore, are compared with results from a 2D axisymmetric cylindrical reactive transport model in which the reactive calcite is present only at the cylinder wall (Figure 3A). The detailed flow field used as input to the modeling is taken from the analytical solution for flow in a cylinder. A typical

modeling result is shown in Figure 3B, where the high flow velocities in the cylindrical pore (approximately $1 \mathrm{~cm} / \mathrm{second}$ in this example) relative to molecular diffusion result in the development of concentration gradients in the pore. Since the

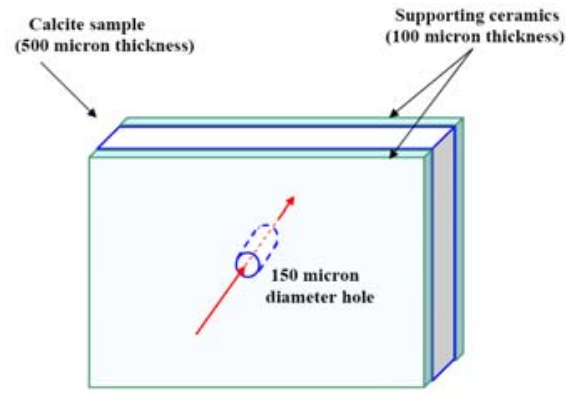

Figure 2: Schematic illustration of microfluidic reactive flow experiment using calcite. rate of calcite dissolution depends on the $\mathrm{pH}$ of the solution, the bulk rate of reaction in the pore differs from what would be obtained if complete mixing were to occur. The result is a flow rateand scale-dependent calcite dissolution rate at the pore scale.

Figure 4 shows a comparison between calcium concentrations in the effluent from the calcite pore with reactive transport modeling results using calcite dissolution rate constants determined in stirred flowthrough reactors and published in the literature. The experiments were conducted at $1 \mathrm{mM}$ ionic strength to avoid the development of gradients in ionic strength that might modify the diffusive flux from the calcite pore wall to the center of the pore. The agreement between the modeling and experiments is good, thus validating the pore scale modeling. 


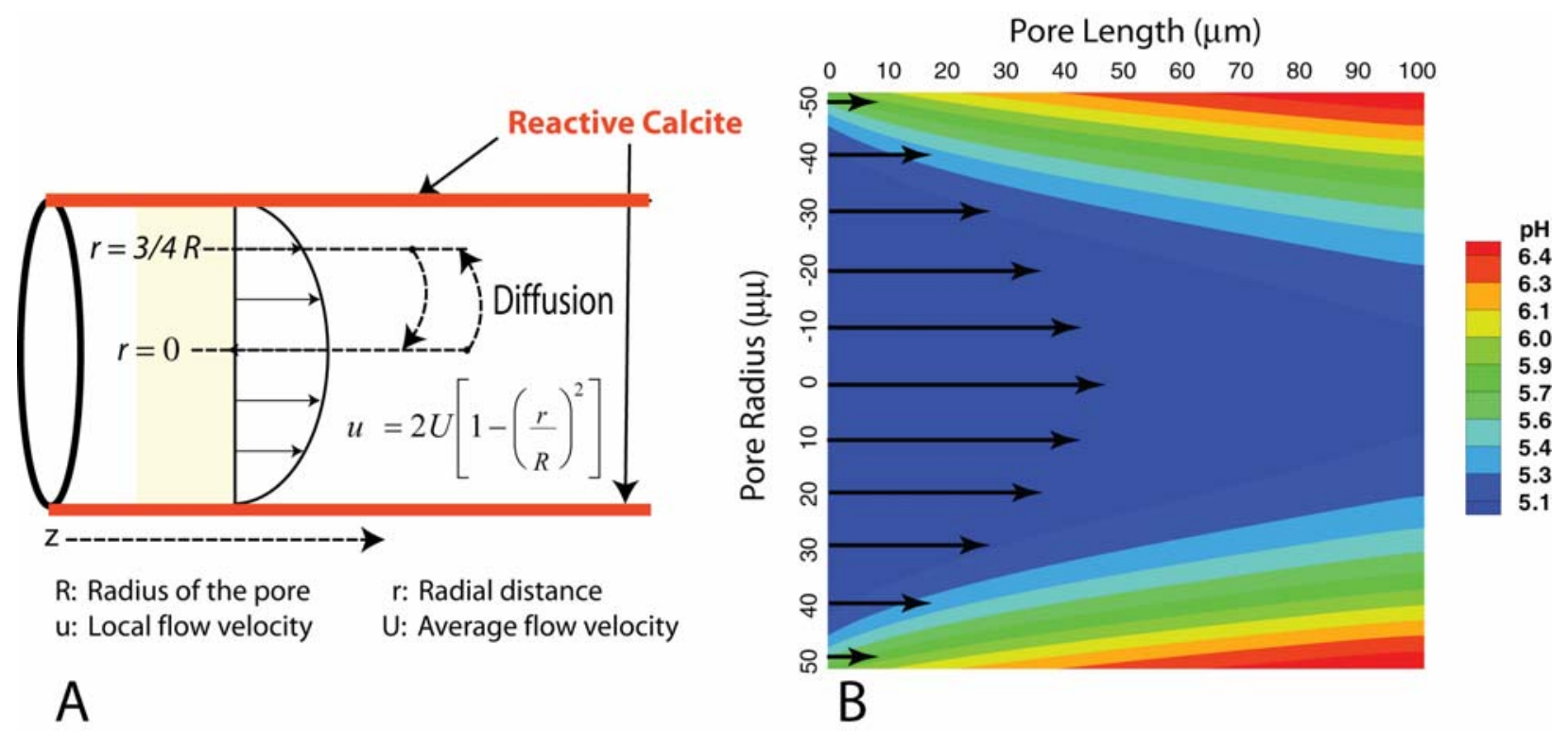

Figure 3: A) Schematic representation of flow and reaction in a single cylindrical pore. B) Contours of $\mathrm{pH}$ for a cylindrical pore within calcite.

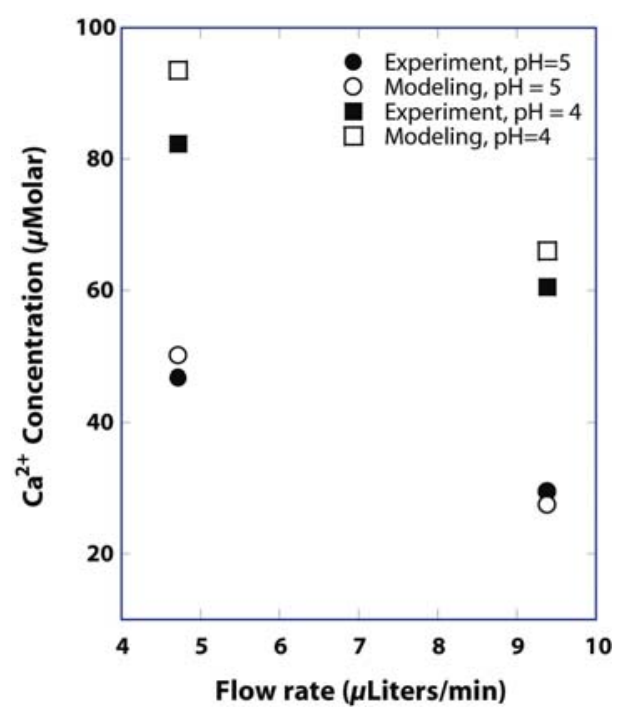

Figure 4: Comparison of results from reactive transport modeling of a single cylindrical pore using kinetic rate constants determined in well-mixed reactor with effluent concentrations from a microfluidic experiment.

\section{Comparison of diffusion modeling using X-ray microtomography with tracer experiments}

A second effort is to use the X-ray microtomography beamline (8.3.2 at the ALS) to create 3D images of the pore structure of weathered basalt at the 2-4 micron scale. This information is then used to establish the correlation between porosity and pore connectivity (which control the percolation and diffusion behavior of the material) and to provide high-resolution pore network maps for input to transport models that quantify the effective diffusivity and permeability of the altered material.

Studies of the weathering of basaltic clasts contained in geomorphic terraces of differing age in Costa Rica indicate that weathering rinds advance linearly as a function of time (Sak et al., 
2004), in contradiction with the expected parabolic time dependence where transport is via diffusion. The linear growth rate of the rinds might be surface-reaction controlled, but the extremely sharp reaction fronts $(<1 \mathrm{~mm})$ seem to argue against this interpretation. As part of our effort to understand the controls on the rates of reaction in natural porous media, we have undertaken $\mu \mathrm{X}$-ray tomographic imaging of the reaction interface separating weathered from fresh rock to elucidate the mechanisms by which the weathering occurs. The tomography carried out on ALS Beamline 8.3.2 indicates that weathering results in the formation of sinuous dissolution channels that enhance both the porosity and its connectivity. This has been confirmed by analyzing the data with the code Percolate from the National Institute of Standards and Technology, which employs a "burning algorithm" to map pore connectivity in 3D samples. A transect across the reaction interface using $25 \mu \mathrm{m}$ microtomographic data blocks was used to map the change in pore connectivity as a function of bulk porosity. The data indicate that there is almost no pore connectivity in the porosity range of $3 \%$ to about $16 \%$ bulk porosity, while between about 16-18\% porosity, a dramatic increase in pore connectivity occurs. Diffusion experiments using a bromide tracer imaged with $\mu$-XRF on Beamline 10.3.2 at the Advanced Light Source are in good agreement with 3D diffusion modeling using data from the X-ray microtomography beamline (Figure 5).

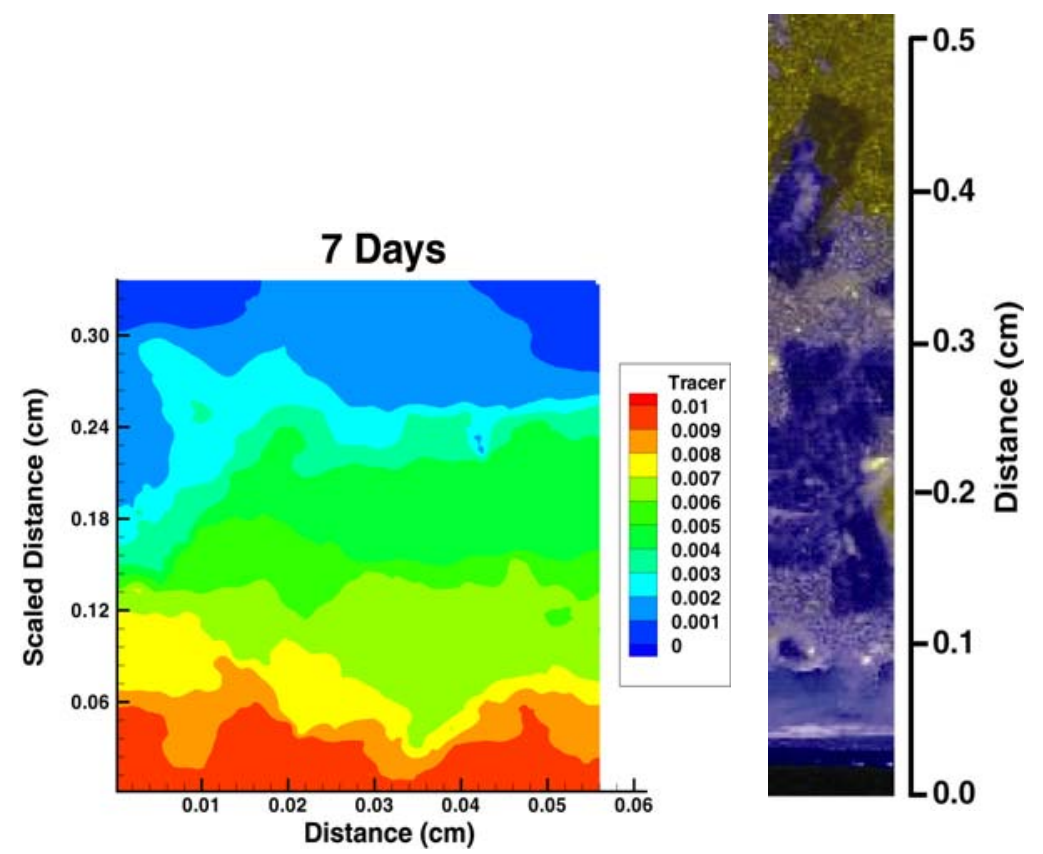

Figure 5: Comparison of 3D reactive transport modeling of tracer diffusion using pore distribution data determined by X-ray microtomography and a bromide tracer experiment on the same basalt using $\mu$-XRF at the Advanced Light Source.

\section{Other Activities}

During the reporting period, Steefel aided in the supervision of one graduate student, Alexis Navarre of Pennsylvania State University. In addition, he co-taught with Peter Lichtner of Los Alamos National Laboratory a three day short course in Reactive Transport Modeling May 11-13 at Pennsylvania State University. The course included a mixture of lectures and laboratory exercises using the code CrunchFlow developed by Steefel. As one-dimensional laboratory exercises included an analysis of the effect of numerical dispersion, a chemical weathering 
problem in spherical coordinates involving granite, and the retardation of ${ }^{137} \mathrm{Cs}$ in various background concentrations of $\mathrm{NaNO}_{3}$ in Hanford sediment. Two dimensional simulations included leaching of copper oxide by a low $\mathrm{pH}$ solution and the evolution of the flow field as a result of reaction-induced porosity and permeability change.

\section{Planned Activities}

Planned activities include new microfluidic experiments involving abiotic and biotic reduction of Fe(III)-oxyhydroxides. In addition, we plan to pre-model and construct 2D thin section style flow through experiments involving chemical heterogeneities to address the issue of the scale dependence of rates at the $\mathrm{mm}$ to $\mathrm{cm}$ scale.

\section{References}

Sak, P.B., Fisher, D.M., Gardner, T.W., Murphy, K., and Brantley, S.L. (2004), Rates of weathering rind formation on Costa Rican basalt: Geochemica et Cosmochemica Acta, v. 68, 1453-1472.

\section{Publications}

\section{Published}

Steefel, C.I, D. DePaolo, and P.C. Lichtner, 2005, Reactive transport modeling: An essential tool and a new research approach for the Earth sciences, Earth and Planetary Science Letters 240: 539-558.

\section{In Preparation}

Li, L., Steefel, C.I., Yang, L., Scaling effects of mineral dissolution rates within single pores. To be submitted to Science.

Steefel, C.I., Navarre-Sitchler, A., Yang, L., A diffusion/percolation threshold model for chemical weathering. 